\title{
Analyse juridique de la liberté de religion dans les entreprises françaises : entre protection du pouvoir de l'employeur et défense de la volonté du salarié Legal Analysis of the Freedom of Religion in French Companies: Between Protecting Employer Power and the Wishes of the Employee

\author{
Análisis jurídico de la libertad de religión en las empresas \\ francesas: entre protección del poder del empleador y defensa \\ de la voluntad del empleado
}

\author{
Philippe Pailot et Diane de Saint-Affrique
}

Volume 74, numéro 2, printemps 2019

URI : https://id.erudit.org/iderudit/1062082ar

DOI : https://doi.org/10.7202/1062082ar

Aller au sommaire du numéro

Éditeur(s)

Département des relations industrielles de l’Université Laval

ISSN

0034-379X (imprimé)

1703-8138 (numérique)

Découvrir la revue

Citer cet article

Pailot, P. \& de Saint-Affrique, D. (2019). Analyse juridique de la liberté de religion dans les entreprises françaises : entre protection du pouvoir de l'employeur et défense de la volonté du salarié. Relations industrielles / Industrial Relations, 74(2), 219-241. https://doi.org/10.7202/1062082ar

\section{Résumé de l'article}

Dans un monde globalisé marqué par le multiculturalisme et le pluralisme confessionnel, la place du religieux cristallise les débats médiatiques, politiques, intellectuels et juridiques dans beaucoup de pays occidentaux. Sur fond de difficultés d'intégration professionnelle de personnes de confession musulmane et de discriminations religieuses au travail, la question du fait religieux s'est invitée dans les entreprises où les manifestations des convictions religieuses se sont diversifiées et complexifiées. L’analyse de ce phénomène social se caractérise par une diversité d'approches et d'angles de vue. Elle illustre tout à la fois la richesse, mais aussi la complexité des enjeux soulevés par les études du lien entre religion et management.

Cet article vise à en proposer une lecture juridique souvent absente dans la littérature en sciences de gestion. Nous chercherons à montrer que la régulation juridique de l'expression des convictions religieuses dans l'entreprise procède d'un champ de tension en équilibre instable entre les normes de droit qui, d'une part, protègent les droits et libertés de religion des salariés et celles, d'autre part, limitent l'exercice du pouvoir de l'employeur. La logique juridique associée à la prise en considération du religieux procède ainsi de la recherche d'une conciliation entre ces deux blocs de droits et libertés. Cette combinaison se nourrit du caractère incertain et équivoque d'un cadre juridique dans lequel les arbitrages entre des prétentions juridiques contradictoires ne procèdent pas d'une application mécanique de règles abstraites et impersonnelles. En effet, il n'existe pas de limites précises et univoques à la manifestation des croyances et des convictions religieuses dans l'entreprise. Leur expression exige toujours des réponses équilibrées.
Tous droits réservés (C Département des relations industrielles de l’Université Laval, 2019
Ce document est protégé par la loi sur le droit d'auteur. L’utilisation des services d'Érudit (y compris la reproduction) est assujettie à sa politique d'utilisation que vous pouvez consulter en ligne. 


\title{
Analyse juridique de la liberté de religion dans les entreprises françaises: entre protection du pouvoir de l'employeur et défense de la volonté du salarié
}

\section{Philippe Pailot et Diane de Saint-Affrique}

\begin{abstract}
Dans un monde globalisé marqué par le multiculturalisme et le pluralisme confessionnel, la place du religieux cristallise les débats médiatiques, politiques, intellectuels et juridiques. Face à cette situation, nous nous intéresserons ici aux champs de tension existant entre les normes de droit qui, d'une part, protègent les droits et libertés de religion des salariés et celles, d'autre part, qui encadrent des libertés économiques et limitent l'exercice du pouvoir de l'employeur. Nous chercherons à montrer que la logique juridique de la prise en considération du religieux dans les entreprises privées procède de la recherche d'une conciliation entre ces deux blocs de droits et libertés qui se nourrit du caractère incertain et équivoque d'un cadre juridique complexe.
\end{abstract}

Mots-clés: fait religieux, liberté de religion, liberté d'entreprendre, principe de neutralité.

\section{Introduction}

L'analyse du fait religieux en entreprise se caractérise par une diversité d'approches qui illustre tout à la fois la richesse, mais aussi la complexité des enjeux soulevés par les études du lien entre religion et management (Weave et Bradley, 2002; Barth, 2012; Galindo et Surply, 2013; Yamane, 2017). Nous avons choisi de l'appréhender sous l'angle juridique. Cette interrogation nous paraît légitime, car sa régulation est indissociable des normes juridiques qui limitent tant le pouvoir de l'employeur que les libertés des salariés. Nous nous intéressons ici aux champs de tension entre les normes de droit qui, d'une part, protègent les droits et libertés de religion des salariés et celles, d'autre part, limitent l'exercice du pouvoir de l'employeur. Nous chercherons à montrer que la gestion de ces champs 
de tension requiert une conciliation entre ces deux blocs de droits et libertés. Cette dernière se nourrit du caractère incertain et équivoque d'un cadre juridique dont l'application exige toujours des réponses équilibrées (Casado, 2017). Ce dernier apparaît une source majeure d'insécurité juridique (Favennec-Héry, 2013) qui rend difficile pour les employeurs et salariés d'apprécier par anticipation les conséquences juridiques de leurs actes.

Notre article se décompose en trois sections. La première aborde le cadre général qui structure la question du fait religieux dans les sociétés de droit privé n'exerçant pas une mission de service public. La deuxième présente les règles de défense du salarié contre les atteintes portées à sa liberté d'expression ou de manifestation de ses convictions religieuses dans l'entreprise. La troisième présente les corps de règles impératives qui protègent le pouvoir et les libertés économiques de l'employeur. Nous conclurons sur l'orientation législative et jurisprudentielle qui tend globalement à renforcer le pouvoir de l'employeur.

\section{Questions autour du fait religieux dans l'entreprise privée}

Le périmètre de notre étude est circonscrit. II n'évoque que le cas des sociétés de droit privé dans le contexte juridique français. Cette délimitation à un type de structure organisationnelle et un territoire permet d'éviter les propos génériques et abstraits totalement décontextualisés. Elle invite à se poser deux questions.

La première porte sur ce qui est régulé. Ce point réclame une triple précision. Tout d'abord, la liberté de religion comporte deux faces irréductibles l'une de I'autre (Guillet, 2003; Gonzalez, 2012; Renucci, 2017): 1- la liberté de croire (apparentée à la liberté d'opinion et de conscience ${ }^{1}$ ) revêt un caractère absolu et échappe donc totalement au contrôle de l'État et, a fortiori, de l'employeur; 2- le droit à la liberté « de manifester ses convictions et ses croyances, individuellement ou collectivement, en public ou en privé, par le culte, l'enseignement, les pratiques et l'accomplissement de rites » (Renucci, 2017: 211) a un caractère relatif. II peut donc faire l'objet de limites dans les conditions prévues par le droit positif. En d'autres termes, la question de la régulation juridique du religieux revient exclusivement à s'intéresser aux limitations des formes d'extériorisation de ce droit sans jamais remettre en cause la liberté de conscience des salariés (Maggi-Germain, 2015).

La deuxième précision concerne le régime de protection renforcée de la liberté de religion. En effet, cette dernière entre à la fois dans le champ des droits et libertés fondamentaux protégés par les normes constitutionnelles et internationales à force supra-législative² (Favoreau et al., 2015; Lécuyer, 2016; Renucci, 2017), et dans celui de la discrimination (Manigot, 2012; Cœuret, Fortis et Duquesne, 2016). Pour illustrer les conséquences de cette double qualification, 
prenons l'exemple de la tenue vestimentaire. En principe, un salarié est libre de la choisir sur son temps et son lieu de travail, sous réserve qu'elle ne soit pas considérée comme incompatible avec ses fonctions et ses conditions de travail. Tout en étant encadrée (CT, L. 1121-1), il s'agit d'une liberté individuelle qui n'entre pas dans la catégorie des libertés fondamentales. Ce point a une conséquence importante en cas de sanction. Un salarié injustement licencié en raison de sa tenue vestimentaire pourrait voir son licenciement requalifié sans cause réelle et sérieuse par un juge, mais il ne serait pas annulés. À l'inverse, une restriction apportée au port d'un vêtement exprimant une appartenance ou des convictions religieuses (foulard, kippa, etc.) peut être une entrave à la liberté de religion (qui est une liberté fondamentale) tout en étant constitutive d'une discrimination (la religion étant l'un des critères de discrimination), ce qui expose l'employeur à des sanctions pénales et civiles (Cœuret, Fortis et Duquesne, 2016). Toute restriction d'expression de la liberté de religion requiert, non seulement de faire preuve d'une plus grande rigueur dans sa justification, mais un salarié injustement licencié pourrait obtenir sa réintégration judiciaire dans l'entreprise 4 .

La troisième précision tient à la diversité des modalités d'expression de la liberté religieuse. Elles n'appellent pas toutes les mêmes analyses juridiques (Antonmattei, 2014), et donc n'induisent pas les mêmes réponses managériales. Les exigences religieuses (demandes de congés pour des fêtes religieuses, de lieux de cultes, demande de prise en compte des restrictions alimentaires, etc.), les comportements religieux (refus de faire certaines choses jugées contraire à des prescriptions religieuses, verbalisation d'une appartenance religieuse, prosélytisme, etc.) ou encore les manifestations d'une appartenance ou de convictions religieuses à travers le port de signes ostensibles (symboles, tenues vestimentaires, apparence physique, etc. qui conduisent à se faire reconnaître immédiatement par son appartenance religieuse) traduisent partiellement cette multiplicité. Face à cette diversité, notre article se focalisera moins sur l'analyse interne de la production normative encadrant ces différentes formes d'expression que sur la rationalité juridique permettant d'appréhender la logique de régulation du religieux dans les sociétés privées.

Ce développement nous amène à notre seconde question, à savoir: Comment régule-t-on le religieux dans l'entreprise? Elle constituera le cœur de notre analyse. Nous chercherons à montrer que la régulation juridique procède de la recherche d'un équilibre subtil intrinsèquement marqué par le sceau de l'incertitude (en raison notamment de la centralité du contrôle du juge) entre, d'une part, la volonté d'un salarié de faire valoir ses convictions religieuses dans l'entreprise et, éventuellement, de les opposer à son employeur et, d'autre part, le pouvoir de ce dernier d'y apporter des restrictions dans les limites incertaines fixées par le droit positif. Cette lecture bipolaire fait écho à la reconnaissance de 
l'entreprise «comme un lieu d'équilibre des pouvoirs et des libertés » (FavennecHéry et Verkindt, 2018) ou encore à l'ambivalence du droit du travail « qui limite le pouvoir de l'employeur tout en le légitimant»(Ray, 2009: 129). Elle revient à considérer le salarié dans sa double qualité de personne physique soumise au principe juridique de subordination ${ }^{5}$, et de personne juridique titulaire de droits pouvant être exercés avec une liberté relative dans le périmètre de ses activités professionnelles (Waquet, Struillou et Pécaut-Rivolier, 2014). Elle reconnaît également la légitimité du pouvoir de l'employeur, de ses libertés économiques, et la nécessité de les encadrer et de les limiter. En ce sens, la gestion de l'expression du religieux oblige à imaginer des équilibres instables et précaires entre des impératifs et des logiques d'acteurs qui peuvent parfois se heurter. Nous verrons que ces deux pôles renvoient à des corps de règles impératives hétérogènes qui visent à protéger, d'une part, les droits et libertés fondamentaux du citoyen-salarié que ce dernier n'abandonne pas en signant son contrat de travail et, d'autre part, le pouvoir et la liberté économique de l'employeur.

\section{La défense de la volonté, la liberté et l'identité du salarié}

Comme nous l'avons évoqué plus haut, la protection juridique contre les atteintes à l'expression des convictions et/ou de l'appartenance religieuses renvoie à deux registres différents de garantie, eux-mêmes attachés à deux corps de règles distincts, à savoir, d'une part, le respect du droit à la liberté de manifestation de ses croyances et, d'autre part, I'application du principe de non-discrimination (Maggi-Germain, 2015).

Cette distinction revient à considérer la religion soit comme un droit attaché à la personne, soit comme une liberté (Maggi-Germain, 2015). Concrètement, il s'agit de se demander si une décision managériale est motivée en raison des convictions religieuses d'un salarié ou de son appartenance à une religion déterminée (état du sujet de droit), ou alors si elle vise à restreindre sa liberté de manifester ses croyances dans l'entreprise (action du sujet de droit). Dans le premier cas, tout traitement défavorable est vu comme une atteinte à sa personne, à la protection de sa dignité humaine et de sa vie privée, voire de son identité. Le cadre juridique applicable est celui du principe de non-discrimination (CT, L. 1132-1). Dans le second cas, il s'agit de savoir si les prescriptions managériales ne remettent pas en cause la liberté de religion des salariés et respectent les exigences de protection contre les atteintes aux droits et aux libertés individuelles et collectives des salariés (CT, L. 1121-1).

En reconnaissant pleinement la proximité entre l'interdiction de discrimination et la protection des libertés (Laronze, 2013; Lyon-Caen, 2013) ${ }^{6}$, cette différenciation a des conséquences analytiques concrètes qui s'ajoutent à la question des 
régimes de protection distincts évoqués dans la première section de cet article. En effet, leur combinaison permet d'appréhender le salarié-citoyen à la fois dans son humanité constitutive et dans son rapport à l'action. D'ailleurs, la non-discrimination religieuse exige la disparition d'une distinction illicite qui se rapporte à un élément constitutif de son identité sociale et/ou personnelle (son appartenance à une religion déterminée et/ou ses convictions religieuses) et à ses raisons d'agir. Elle s'adresse uniquement à des groupes sociaux dont les membres sont susceptibles d'être stigmatisés en raison de caractéristiques subies (sexe, origine ethnique, etc.) ou de choix effectués au nom de l'exercice de certaines libertés fondamentales (convictions religieuses, opinions politiques, etc.). En garantissant à l'individu la libre disposition de son esprit (Manigot, 2012), la non-discrimination vise à prohiber les atteintes à la personne dans son ontologie (Lyon-Caen, 2013) appréhendée au prisme de qualités singularisées (Porta, 2013). La liberté de religion met, quant à elle, avant tout l'accent sur le libre arbitre (au détriment de "l'identité ») et la liberté de choix (elle relève en premier lieu de la liberté de pensée et de conscience de chacun) dans lesquels la religion, et ses exigences, sont considérées comme choisies et non subies (Calvès, 2018). Les restrictions apportées à son expression concernent les attitudes et comportements du salarié qui s'expriment dans la sphère sociale par des choix (port de signes religieux, etc.) justifiés au nom de son pouvoir d'autodétermination (donc de sa liberté) opposable à son employeur et aux autres salariés (Sachs, 2012).

Nous allons maintenant revenir sur les contours de ces deux règles impératives qui obligent l'employeur à devoir justifier objectivement l'essentiel des décisions individuelles et/ou collectives qu'il prend en matière de restriction de la liberté de religion dans l'entreprise.

\section{Le principe de non-discrimination}

Le principe de non-discrimination renvoie à l'interdiction d'arrêter une décision ou d'établir une distinction entre des personnes ou des groupes de personnes sur le fondement de critères interdits par le droit (Manigot, 2012; Favennec-Héry et Verkindt, 2018). II ne s'oppose pas à la nécessité managériale d'établir des distinctions dans le traitement des individus qui seraient justifiées par la liberté contractuelle (Porta, 2013). Pour établir des différences de traitement, il interdit seulement la prise en compte de certains critères de différenciation sociale définis par le législateur comme discriminatoires (Porta, 2013; Hennette-Vauchez et Wolmark, 2016). En droit, c'est le pouvoir législatif qui fixe la liste limitative des motifs de différenciation prohibés ${ }^{7}$ en marquant "le périmètre au-delà duquel un traitement inégal s'opère en toute légalité » (Bereni et Chappe, 2011: 13). Au gré des multiples réformes successives, l'allongement de cette liste a conduit à étendre considérablement le périmètre du champ de la discrimination en re- 
groupant dans un même texte des motifs disparates qui protègent des valeurs ne revêtant pas la même importance et dont la multiplication tend à affaiblir le concept originel (Manigot, 2012).

Dans le contexte des relations du travail salarié, la répression de la discrimination fait l'objet d'une double approche, à la fois par le Code du travail (L. 1132-1) et par le Code pénal (L. 225-1). Ce dernier pose le critère de l'appartenance ou de la non-appartenance, vraie ou supposée, à une religion déterminée. Le Code $d u$ travail retient celui des convictions religieuses. Cette distinction revient à différencier l'état du sujet de droit (appartenance réelle ou supposée à une religion) de son action (expression de ses convictions religieuses) (Pagnerre, 2017). Audelà de l'absence de délimitation sémantique et théorique précise, la notion de conviction ne s'apprécie pas au prisme exclusif de la religion ${ }^{8}$ (Calvès, 2018). Ce terme "s'applique à des vues atteignant un certain degré de force, de sérieux, de cohérence et d'importance » (CEDH, 25 fév. 1982, n 7511/6; 7743/76) susceptibles de s'incarner dans des exigences éthiques, voire un mode de vie.

La connotation péjorative associée à la discrimination au nom d'exigences abstraites de justice sociale (particulièrement marquée dans les sciences sociales) tend à faire oublier que sa prohibition n'est, en droit, en rien absolue. Si le langage juridique ne monopolise pas le sens donné à la notion de discrimination (Bereni et Chappe, 2011), le droit l'appréhende de façon relative. II admet que certaines différences de traitement potentiellement discriminatoires puissent déroger au principe de non-discrimination et être, dans certains cas, légitimes et justifiées, en rendant ainsi les dispositions sanctionnatrices inapplicables (Moreau, 2002; Cœuret, Fortis et Duquesne, 2016). Ainsi, le principe de nondiscrimination ne fait pas «obstacle aux différences de traitement, lorsqu'elles répondent à une exigence professionnelle essentielle et déterminante et pour autant que l'objectif soit légitime et l'exigence proportionnée » (CT, L. 1133-1). Pour être autorisées, les distinctions fondées sur un ou plusieurs critères discriminatoires doivent répondre à une triple exigence stricte et cumulative et ce, sous le contrôle du juge. Tout d'abord, l'évocation d'un critère (une caractéristique de l'être ou de l'agir d'une personne) doit être une exigence professionnelle (en lien direct et nécessaire avec la situation professionnelle et le contexte de travail sans être l'expression de l'arbitraire patronal), essentielle (la règle imposée doit être essentielle à la réalisation de la prestation de travail) et déterminante (existence d'une incompatibilité objective, pertinente et matériellement vérifiable de l'expression du fait religieux avec l'emploi ou les fonctions occupées). Ensuite, les différences de traitement doivent poursuivre objectivement et raisonnablement un but légitime comme la protection de la santé ou de la sécurité du salarié (François, 2013), le maintien de sa capacité à réaliser son travail (Manigot, 2012) ou encore certaines contraintes vestimentaires comme, par exemple, le port d'un 
uniforme dans certains métiers ou certaines fonctions (Schütz, 2018). Enfin, I'exigence doit être proportionnée, c'est-à-dire qu'il doit exister un rapport, une adéquation entre les moyens utilisés par l'employeur et le but qu'il vise (conciliation entre les nécessités du bon fonctionnement de l'entreprise et la protection des droits de la personne et des libertés individuelles et collectives des salariés). II convient notamment de s'assurer qu'une mesure moins restrictive ne permettrait pas de parvenir au même objectif.

Sans être laissée à la libre appréciation des entreprises, une rupture d'égalité en raison de l'expression de convictions religieuses dans l'espace professionnel peut donc être tout à fait légitime en droit. Loin d'être un facteur de protection unilatérale du salarié dans l'expression de ses droits et libertés, le droit de la discrimination permet également de protéger et de contrôler l'employeur dans ses droits et libertés, ainsi que dans l'exercice de son pouvoir.

\section{La protection des libertés du salarié}

Notion particulièrement complexe (Fabre-Magnan, 2018), la liberté peut se définir, sur le plan ontologique, comme «un pouvoir d'autodétermination, en vertu duquel I'homme choisit lui-même son comportement personnel » (Lebreton, 2012 : 619). En droit, toute liberté est, par définition, à la fois positive (possibilité de manifester ses convictions religieuses) et négative (possibilité de ne pas le faire) (Fabre-Magnan, 2018). Sphère d'action qui échappe partiellement au pouvoir de l'employeur, les restrictions de la liberté de religion sont encadrées pour l'essentiel par l'article L. 1121-1 du Code du travail: "Nul ne peut apporter aux droits des personnes et aux libertés individuelles et collectives des restrictions qui ne seraient pas justifiées par la nature de la tâche à accomplir ni proportionnées au but recherché ». Cet article a un champ d'application et une portée bien plus vastes que celui de la seule liberté religieuse. Il permet d'embrasser les restrictions individuelles et collectives de toutes natures en s'intéressant à leurs effets sur les droits et libertés des salariés (Fabre, 2010). Dans le cadre de l'exécution de son contrat de travail, le salarié conserve ainsi les droits et libertés liés à sa personnalité juridique qui peuvent être réduits, mais jamais supprimés: «même en exécutant loyalement son travail, sa vie personnelle n'est pas totalement abolie et un usage modéré de sa liberté subsiste dans la subordination », nous disent Waquet, Struillon et Pécaut-Rivolier (2014: 234). En d'autres termes, même dans la limitation partielle de sa liberté inséparable de l'application du principe juridique de subordination, «le salarié reste un homme libre » (Waquet, Struillon et Pécaut-Rivolier, 2014: 234).

A contrario, I'article L. 1121-1 du Code du travail légitime la légalité de certaines restrictions apportées par l'employeur dès lors qu'elles répondent à une double exigence. Elles doivent être justifiées par les impératifs du travail (appré- 
ciés au regard de la nature des tâches à accomplir et non de manière générale et abstraite) et respecter le principe de proportionnalité (mesure de l'adéquation entre les moyens déployés et la finalité poursuivie, réduction des atteintes au strict minimum en excluant toute forme de restriction permanente, absolue et générale applicables à tous les postes et/ou tous les salariés, sans distinction de fonction ou de mission). De manière générale, ces règles apparaissent plus souples que le cadre juridique applicable au principe de non-discrimination. Comme pour ce dernier, la protection de libertés individuelles et collectives des salariés n'est pas un principe absolu et leurs restrictions apparaissent tout à fait légitimes dans des conditions somme toute restrictives.

En conclusion de cette deuxième section, nous avons pu voir que les fondements de la protection de l'expression des convictions religieuses du salarié dans l'espace de travail ne sont nullement absolus. L'indifférence à sa religion cesse lorsque les impératifs de sa foi ont des répercussions sur la réalisation des prestations qui lui sont demandées, ou encore face aux exigences du bon fonctionnement de l'entreprise (Manigot, 2012). Ces formes de restrictions de la liberté de religion sont complétées par un ensemble de dispositions spécifiquement dédiées à la protection du pouvoir et des libertés économiques de l'employeur.

\section{La protection du pouvoir et des libertés économiques de l'employeur}

Le pouvoir de l'employeur est un phénomène protéiforme (Fabre, 2010) dont l'exercice est encadré par de nombreuses règles législatives et jurisprudentielles. II se trouve conforté par la reconnaissance constitutionnelle de ses libertés fondamentales, et notamment celle d'entreprendre et de choisir ses collaborateurs (Mazeaud, 2016; Favennec-Héry et Verkindt, 2018). Pour ce qui nous intéresse ici, c'est essentiellement la force obligatoire du contrat, la liberté d'entreprendre et le principe de neutralité qui légitiment les restrictions apportées par l'employeur à l'expression de la liberté religieuse des salariés dans l'entreprise.

\section{De la force obligatoire du contrat au pouvoir juridique de l'employeur}

Toutes choses restant égales par ailleurs, les normes juridiques tendent à circonscrire l'expression de la volonté du salarié dans le champ contractuel (Gratton, 2012). Ces restrictions sont en partie la conséquence du lien de subordination juridique (limité au temps et au lieu de travail) consubstantiel au contrat de travail. Ce principe légitime l'exercice du pouvoir de l'employeur dans ses dimensions réglementaire (ou normative), disciplinaire et de direction (Fabre, 2010). D'une manière générale, le contrat ayant une force obligatoire ${ }^{9}$, la demande de 
son exécution n'appelle pas, en soi, de justification. Un salarié ne peut donc pas évoquer son appartenance à une religion donnée, ses convictions ou des obligations religieuses pour refuser d'effectuer la prestation de travail qu'il s'est engagé contractuellement de fournir. Par contrat, tout salarié est donc tenu de mettre sa force de travail à la disposition de son employeur et de se conformer à ses ordres et directives, dès lors que ceux-ci sont relatifs à sa prestation de travail et restent dans le champ de ses attributions contractuelles (Casado, 2017; Auzero, Baugard et Dockès, 2018). Si le refus du salarié d'effectuer ce que lui demande son employeur peut être légitime dans des cas restrictifs (Lokiec, 2012), la volonté du salarié ne peut pas se comprendre dans un rapport équilibré avec le pouvoir de direction. Ainsi, un salarié qui refuserait de travailler sous la direction d'une personne en raison de son sexe, son appartenance religieuse ou autres critères, qui quitterait délibérément son poste sans l'accord de sa hiérarchie pendant son temps de travail effectif pour se livrer à des activités cultuelles, ou encore qui utiliserait sans autorisation les locaux de l'entreprise à des fins religieuses, s'exposerait à des sanctions disciplinaires. Dans la même veine, la liberté d'expression de la religion ne saurait être avancée par un salarié pour placer de manière délibérée son employeur en situation de ne pouvoir remplir ses obligations comme, par exemple, celles qui existent en matière de santé et de sécurité au travail (François, 2013; Adam et al., 2016). Ainsi, le refus pour des raisons religieuses d'une visite médicale, d'une vaccination et du port d'un vêtement de sécurité rendus obligatoires par la législation ou une autre règle normative constitue une faute pouvant justifier un licenciement.

En restant dans le champ du pouvoir de direction de l'employeur, l'organisation de l'entreprise et du travail reste à l'appréciation de ce dernier (Mazeaud, 2016). II n'est pas tenu de modifier l'organisation du travail pour rendre possible l'exécution d'obligations religieuses. À ce titre, les individus ou les groupes qui transgressent sciemment les règles de l'organisation en vue de les adapter à des prescriptions religieuses ou d'imposer ces dernières au fonctionnement de I'organisation s'exposent clairement à des sanctions disciplinaires. Sauf clauses contractuelles particulières (Gardes, 2017), les salariés ne peuvent pas exiger un traitement particulier en rapport avec leur religion comme, par exemple, des aménagements d'horaires pour certains rites, l'autorisation d'absence pour certaines fêtes, la création de lieux de prière dans l'entreprise, le respect des interdits ou exigences alimentaires. Sur ce plan, la liberté religieuse est traitée comme les autres libertés. Elle ne peut pas donner lieu à un traitement plus favorable en raison de son caractère fondamental. Dans le respect du principe de bonne foi contractuelle, il existe une grande latitude de l'employeur pour accorder ou refuser une requête dès lors que sa décision est fondée sur des impératifs tenant à l'organisation du travail et de la production (Gaudu, 2010). Toutefois, son refus ne peut pas se justifier en raison du caractère religieux de la 
demande, ce qui le placerait dans le champ de la discrimination directe ou indirecte. Dans la même veine, l'employeur reste le garant des droits et libertés de chacun dans l'entreprise. Si l'appréciation de sa preuve est rigoureuse, il peut donc sanctionner disciplinairement le prosélytisme (notamment) religieux ${ }^{10}$ qui porterait atteinte à la liberté de conscience des autres salariés ou, plus généralement, perturberait le fonctionnement normal de l'entreprise (Gardes, 2017). Il existe un deuxième argument de légitimation des restrictions du droit des salariés à exprimer leurs convictions religieuses dans l'entreprise, à savoir la liberté économique.

\section{Liberté économique et pouvoir de gestion de l'employeur}

La jurisprudence nationale admet depuis longtemps que les impératifs de fonctionnement de l'entreprise et/ou la protection de ses intérêts légitimes puissent être invoqués, à titre défensif, pour restreindre les droits et libertés des salariés (Fabre, 2010). Comme le note cette auteure, l'exigence de légitimité (qui situe I'analyse sur le terrain des valeurs) ne se confond pas avec l'objectivité (qui invite à vérifier que la raison évoquée par l'employeur est bien celle pour laquelle il a véritablement agi). Sur ce point, la Cour de justice de l'Union européenne (CJUE, 14 mars 2017, n C-157/15) considère que le souhait d'un employeur d'afficher, dans les relations avec les clients tant publics que privés, « une politique de neutralité politique, philosophique ou religieuse doit être considérée comme légitime ». La juridiction européenne ajoute un élément déterminant. Elle considère que cette exigence de neutralité «se rapporte à la liberté d'entreprise» (CJUE, 14 mars 2017, n C-157/15), reconnue par la Chartre des droits fondamentaux de l'Union européenne (art. 16) qui «a une force juridique contraignante dans I'Union» (Favennec-Héry, 2013: 13). La légitimation par CJUE du principe de neutralité par la liberté d'entreprise (constitution et gestion d'une entreprise) revient à dire qu'une liberté économique possède un caractère fondamental qui peut être opposé aux États, mais aussi aux droits et libertés fondamentaux du salarié. Cette consécration de la liberté économique comme droit fondamental n'est pas propre au droit européen. Elle se retrouve en droit interne à travers la notion de liberté d'entreprendre.

Ayant une valeur constitutionnelle largement consacrée depuis le début des années 1980 (Drago et Lombart, 2003), «la liberté d'entreprendre est essentiellement la liberté du chef d'entreprise de ne pas se voir imposer des contraintes excessives dans la sphère propre de son pouvoir de gestion» (Lombard, 2012: 626), c'est-à-dire qu'elle consacre, pour l'essentiel, l'indépendance ou la liberté de gestion de l'entreprise (d'Avout, 2014; Lussart, 2015). Cette consolidation de la liberté d'entreprendre au rang de liberté fondamentale lui permet de bénéficier «des garanties juridictionnelles propres à tout droit fondamental» 
(Altwegg-Boussac, 2011 : 248), et, notamment, de faire obstacle à d'autres droits et libertés fondamentaux (Champeil-Desplats, 2007). Ce point est important pour notre propos.

La recherche d'équilibre entre le pouvoir de l'employeur et la volonté ou la liberté du salarié de manifester ses convictions religieuses procède donc d'une confrontation entre deux droits-libertés fondamentaux, à savoir la liberté d'entreprendre et le droit à la liberté de religion, dont aucun n'a un caractère absolu. Ces deux libertés ayant une égale prétention à la fondamentalité, chacune d'entre elles trouve une limite nécessaire dans le respect de l'autre. Les limitations apportées ne doivent pas avoir pour conséquence d'en dénaturer I'une ou l'autre (Favoreau et al., 2015) et les atteintes ne doivent pas se révéler disproportionnées ou manifestement excessives au regard de l'objectif poursuivi (Lyon-Caen, 2002).

En d'autres termes, dans le conflit ou la ligne de tension entre les valeurs contradictoires de la logique marchande et des droits de l'Homme, les libertés économiques de l'employeur ne sont pas structurellement subordonnées aux libertés, même fondamentales, du salarié. Ces deux libertés ne sont a priori pas hiérarchisées. II s'agit plutôt de maintenir une conciliation, un " juste équilibre »"11 entre des principes constitutionnels contradictoires, afin de respecter le subtil équilibre voulu et établi par le législateur. L'équilibre des intérêts en présence sera plus ou moins proportionné selon les activités considérées et les droits et les libertés en cause. En suivant les motivations de la CJEU (14 mars 2017, n C-157/15), la liberté économique peut prévaloir sur la volonté des salariés et être invoquée pour justifier des restrictions à leurs libertés et leurs droits, mêmes fondamentaux. Elle devient alors une "force privative de liberté des individus » (Champeil-Desplats, 2007). Dans les modes de résolution des conflits entre les libertés économiques (logique marchande) et les droits de l'Homme (logique humaniste), le rôle du juge, en référence au contrôle de proportionnalité au regard de l'objectif poursuivi, devient alors central en cas d'échec de toute forme de conciliation.

Selon Pagnerre (2017), la décision de la CJUE a une portée considérable, car elle transforme la liberté d'entreprise «en objectif légitime présumé de manière quasi irréfutable». Une liberté fondamentale du salarié semble, en effet, devoir céder sur l'intérêt économique de l'entreprise. Cette décision s'inscrit en cohérence avec le mouvement amorcé, tant en droit européen (Hervieu, 2011) qu'en droit interne (Altwegg-Boussac, 2011), qui a largement renforcé la protection de la liberté d'entreprendre dans sa confrontation aux droits de l'Homme. Dès lors, la finalité économique de l'entreprise peut logiquement être vue, sous certaines conditions, comme un motif légitime de restriction du droit à la liberté d'exprimer ses convictions, notamment religieuses. L'inverse re- 
viendrait à refuser a priori une légitimité aux libertés économiques qui devraient être systématiquement subordonnées à la "religion des droits de l'Homme» (Lacroix et Pranchère, 2016). Comme le suggère d'Avout (2014), cette appréciation conduirait à créer une rupture d'égalité de traitement entre les citoyens en considérant, par principe, en termes moins favorables les employeurs que les salariés-citoyens sans autre justification que morale, philosophique, voire idéologique. Nous allons maintenant présenter le troisième et dernier argument de justification de la restriction de la liberté de religion des salariés, à savoir le principe de neutralité.

\section{Pouvoir normatif et principe de neutralité dans l'entreprise}

L'évocation d'un principe de neutralité pour les entreprises privées pourrait surprendre tant celui-ci est une notion propre au droit public (Philip-Gay, 2016). II est, en effet, étroitement associé, voire consubstantiel, à celui de laïcité. Principe constitutionnel d'organisation de l'État (neutralité qui l'oblige à se tenir à égale distance des religions, respect de toutes les croyances, égalité de tous les citoyens devant la loi sans distinction de religion) et de la République (reconnaissance et financement d'aucun culte, garantie de la liberté d'exercice des cultes), la laïcité consacre la neutralité, voire la sécularisation, tant de l'État que des services publics (Gonzalez, 2006; Zuber, 2017). Appliqué à l'entreprise, le principe de neutralité présente deux différences majeures avec cette conception. Tout d'abord, il n'est pas associé au principe de laïcité (Pagnerre, 2016; Gardes, 2017), mais résulte du pouvoir normatif (réglementaire) de l'employeur à travers le règlement intérieur (désormais RI) ${ }^{12}$. Ensuite, ses conditions d'application ont une portée beaucoup moins restrictive qu'en droit public où la neutralité présente un caractère absolu.

Pour éviter certaines confusions, rappelons que le principe de laïcité ne s'impose qu'à la sphère publique. II n'est en aucune manière applicable aux salariés des entreprises de droit privé qui ne gèrent pas un service public (Morena, 2015). Les fondements juridiques qui justifient l'usage de la notion de neutralité dans I'entreprise sont d'une autre nature. En droit interne, ce principe a été introduit par la voie législative et transposé dans I'article L. 1321-2-1 du Code du travail. Ce dernier décrète que:

Le règlement intérieur peut contenir des dispositions inscrivant le principe de neutralité et restreignant la manifestation des convictions des salariés si ces restrictions sont justifiées par l'exercice d'autres libertés et droits fondamentaux ou par les nécessités du bon fonctionnement de l'entreprise et si elles sont proportionnées au but recherché.

Comme le précise Pagnerre (2016), ce texte identifie la source, le contenu, la justification et les conditions du principe de neutralité. 


\section{Source}

La source est le RI qui est à la fois un acte unilatéral s'imposant à tous (y compris à l'employeur) et la source du pouvoir disciplinaire de ce dernier (Fabre, 2010). Ne résultant pas d'une obligation impérative, I'application du principe de neutralité est donc contingente à chaque entreprise disposant d'un $\mathrm{RI}^{13}$. En respectant les formalités de dépôt et de publicité, c'est l'employeur, et seulement lui, qui décide de l'application ou non du principe de neutralité et définit ce qu'il entend par la manifestation des convictions. L'inscription de ce principe dans le RI signifie aussi que sa violation par un salarié peut justifier une sanction disciplinaire en cas de non-respect des préconisations qu'il comporte. En conformité avec les dispositions du Code du travail (CT, L. 1321-5), la Cour de cassation précise que la clause de neutralité peut être inscrite «dans une note de service soumise aux mêmes dispositions que le règlement intérieur» (CC, soc. 22 novembre 2017, $\left.n^{\circ} 13-19.855\right)$. En revanche, la Cour précise, en toute logique, qu'elle ne peut pas résulter d'une consigne ou d'un ordre oral. Elle n'aurait alors aucune valeur légale et ne serait pas opposable au salarié.

\section{Contenu}

Le contenu porte sur l'inscription d'un principe de neutralité dans le RI et la restriction de la manifestation de convictions qui peuvent être de natures diverses: philosophiques, politiques, morales, religieuses ou autres. Cette position est cohérente avec celle de la CJUE (14 mars 2017, n C-157/15) qui évoque la question de la neutralité sous l'angle du port de signes visibles de convictions politiques, philosophiques ou religieuses. La politique de neutralité doit s'appliquer «de manière générale et indifférenciée», tout en étant « véritablement poursuivie de manière cohérente et systématique » (CJUE, 14 mars 2017, n C-157/15; CC, soc. 22 novembre 2017, n¹3-19.855). A contrario, elle ne peut pas viser uniquement un signe religieux déterminé sous peine de rentrer dans le champ de la discrimination directe ou indirecte fondée sur la seule religion ou les convictions religieuses.

\section{Justification}

Le principe de neutralité se justifie par la volonté de protéger d'autres libertés et droits fondamentaux (notamment la liberté d'entreprise - cf. supra) ou les nécessités du fonctionnement de l'entreprise. Le premier point est tout à fait conforme à la Convention européenne des droits de l'Homme (art. 9-2) ${ }^{14}$. Le droit à la liberté religieuse des uns doit s'opérer dans le respect des libertés fondamentales d'autrui, notamment celles de conscience et d'opinion. Les mesures restrictives prévues par le RI doivent pouvoir permettre à chacun de se sentir respecté dans sa sensibilité particulière. 
Le second critère de justification du principe de neutralité renvoie aux nécessités du bon fonctionnement de l'entreprise. La liberté de religion doit donc être conciliée avec les intérêts de l'entreprise. Relevant de l'appréciation de l'employeur, cet argument conduit à justifier une restriction de liberté par des motifs économiques et non en vertu de l'application d'une valeur ou d'un principe républicain: «la volonté d'afficher, dans les relations avec les clients tant publics que privés, une politique de neutralité politique, philosophique ou religieuse doit être considérée comme légitime » (CJUE, 14 mars 2017, n C-157/15). En cohérence avec le contrôle de proportionnalité, le périmètre d'une clause de neutralité n'en reste pas moins restrictif. II ne peut s'appliquer «qu'aux salariés se trouvant en contact avec les clients » (CC, soc. 22 novembre 2017, n¹3-19.855) ou, par extension, à toutes personnes ayant une fonction de représentation de l'entreprise vis-à-vis des parties prenantes externes.

\section{Conditions de légalité}

Les conditions du principe de neutralité ne remettent donc pas en cause les règles de justification et de proportionnalité familières aux juristes. «Tout en tenant compte des contraintes inhérentes à l'entreprise et sans que celle-ci ait à subir une charge supplémentaire », l'entreprise doit apprécier "s'il lui est possible de proposer» au salarié « un poste de travail n'impliquant pas de contact visuel avec ces clients, plutôt que de procéder à son licenciement » (CC, soc. n¹3-19.855, 22 novembre 2017). C'est-à-dire que, dès lors qu'elles sont justifiées, les atteintes doivent être limitées au strict nécessaire (pour un temps, des fonctions et une activité précise) et donner lieu à des «accommodements raisonnables» (notion propre au droit anglo-saxon). Elles ne peuvent pas être permanentes et générales. Dans la même veine, la CJUE (14 mars 2017, n C-157/15) évoque également une interdiction de ports de signes qui «se limite au strict nécessaire » pour atteindre le but poursuivi — en l'occurrence la relation avec les clients.

En droit privé, la neutralité permet à l'employeur de prohiber indifféremment le port de signes et les manifestations visibles de convictions politiques, philosophiques et/ou religieuses à des catégories précises de salariés (ceux qui sont en contact avec la clientèle) et ce, dans le respect du principe de l'égalité de traitement. Tous les salariés placés dans des situations comparables doivent être traités de manière identique sans que l'employeur puisse établir de distinction selon la nature de leurs convictions exprimées dans l'entreprise. Ce principe traduit une exigence de neutralité des personnes privées et de leurs relations dans un idéal de dépassement des identités et des modes d'expression publics dans les relations de travail. II donne un pouvoir à l'employeur pour limiter plus facilement l'expression desdites convictions des salariés dans l'objectif de préserver un équilibre entre les droits-libertés du salarié citoyen et la protection des intérêts de l'entre- 
prise. Le principe de neutralité renforce-t-il les restrictions qui existaient quant à l'expression des convictions des salariés dans l'entreprise avant son introduction dans le Code du travail? Se fondant sur une règle générale et permanente posée par écrit qui offre des garanties procédurales, il permet à l'employeur d'imposer unilatéralement aux salariés d'une entreprise privée une obligation de neutralité dont la violation les expose à un licenciement disciplinaire (Gardes, 2017). En ce sens, il nous semble renforcer la sécurité juridique des entreprises au détriment de la liberté des salariés.

\section{Conclusion}

L'expression de la liberté religieuse dans l'entreprise privée exclut d'emblée toute interdiction générale et absolue, applicable à tous les salariés, sans distinction de profession ou de mission. Ce droit fondamental peut être tout au plus limité ou restreint sans pouvoir être supprimé. Il doit être concilié avec d'autres impératifs. L'appréciation de la légitimité de restriction de la liberté d'expression religieuse dans l'espace de travail est une question complexe où des considérations éthiques, morales, idéologiques et juridiques peuvent s'entrechoquer parfois de manière insidieuse. Les propos de Yannick Pagnerre (2016: 880) reflètent bien cette difficulté: "Ô combien il est difficile, en France, cinquième pays le plus athée au monde, de parler du fait religieux dans l'entreprise sans déchaîner les passions ». En raison son histoire institutionnelle, politique, juridique et religieuse (dont la présentation dépasserait largement le cadre de cet article), la France a, au-delà de la mise en exergue hagiographique d'une laïcité mythifiée qui falsifie largement les conditions historiques réelles de sa construction (Poulat, 2003; Lalouette, 2005; Baudérot, 2015; Zuber, 2017), présente en matière de religion, d'importantes singularités qui surprennent beaucoup hors de nos frontières (Gherardi, 2017). Elle a notamment une approche plus conflictuelle et idéologique des questions religieuses que les autres États-Nations occidentaux et une réticence plus marquée à l'expression publique visible des appartenances religieuses (Willaime, 2005; Bobineau, 2012; Machelon, 2015). À ce contexte social-historique singulier s'ajoute, en France, l'existence de préjugés très négatifs à l'encontre des personnes d'origine maghrébine et de confession musulmane (Wagner, Tisserant et Bourhis, 2013), et plus largement vis-à-vis de la religion musulmane (Tiberj, 2015) ${ }^{15}$. Face aux demandes de prise en compte du fait religieux dans le management et l'organisation, les réponses des entreprises ne sont pas pour autant monolithiques. Elles restent variables et peuvent osciller, par exemple, entre les voies du refus, de la tolérance ou de l'accommodement (Galindo et Zannad, 2014).

Nous avons cherché à montrer que la régulation du religieux dans l'entreprise ne peut pas s'appuyer sur des hiérarchies claires entre les droits et libertés du salarié et ceux de l'employeur. Ceux-ci doivent être combinés ou conciliés afin de 
respecter l'équilibre subtil voulu par le législateur entre l'intérêt de l'entreprise, le pouvoir de l'employeur et les droits fondamentaux des salariés. Dans cet art difficile des conciliations (Adam et al., 2016), les dernières inflexions jurisprudentielles et législatives donnent le sentiment que le balancier penche plutôt en faveur de l'employeur tant ce dernier pourra, avec le principe de neutralité, délimiter unilatéralement les contours du périmètre et des modalités d'expression des droits et libertés des salariés en matière de convictions religieuses. Cette évolution peut être lue comme un facteur de renforcement de la sécurité juridique des entreprises dans le domaine des droits et libertés fondamentaux qui reste l'un des plus importants facteurs d'insécurité juridique en droit du travail (Favennec-Héry, 2013). On peut y voir également un recul des droits et libertés fondamentaux des salariés, au profit de l'intérêt de l'entreprise (Gardes, 2017). L'appareil normatif n'est pas pour autant libéral et ne cautionne pas toutes les demandes subjectives de l'employeur. Les restrictions apportées à la liberté de religion des salariés restent tout de même étroites, fortement encadrées et soumises au contrôle permanent du juge. En ce sens, l'évolution du droit du religieux dans l'entreprise ne consacre pas une déclinaison singulière de la «nouvelle laïcité » (Hennette Vauchez et Valentin, 2014), un renforcement de la «laïcité de contrôle » (Zuber, 2017) ou encore «l'extension, par petites touches, du domaine de la laïcité » (Gherardi, 2017: 85) dans le droit de l'entreprise privée. La frontière entre le respect de la liberté religieuse du salarié et la nécessité de laisser à l'employeur la possibilité d'exercer son pouvoir de direction reste fragile et largement incertaine. La gestion juridique du fait religieux requiert, et continuera d'exiger, un pilotage fin qui ne saurait remettre en cause le principe de défense de la liberté religieuse sise au cœur du pacte républicain dans lequel la liberté est la règle et l'interdiction l'exception.

\section{Notes}

1 La liberté de conscience est entendue «comme le droit à déterminer librement en son for intérieur ses croyances et convictions propres, qu'elles soient religieuses ou non » (Guillet, 2003: 183).

2 Au-delà de sources du droit interne, la liberté de religion est protégée par le droit international (art. 18 et 19 de la Déclaration universelle des droits de l'Homme (DUDH), Pacte international relatif aux droits civils et politiques, art. 14 de la Convention internationale des droits de l'enfant, etc.) et européen (Convention européenne de sauvegarde des droits de l'Homme et des libertés fondamentales (art. 9), Chartre des droits fondamentaux de l'Union européenneUE (art. 10), Directive 2000/78/CE du 27 novembre 2000 (art. 1 et 10), etc.

3 En effet, un juge ne peut pas, en l'absence de disposition le prévoyant et à défaut de violation d'une liberté fondamentale, annuler un licenciement (Cass., soc., 13 mars 2001, n 99-45.735P).

4 En effet, tout acte discriminatoire est nul en droit, c'est-à-dire que l'employeur est tenu de remettre les choses en l'état où elles étaient avant l'acte illicite. 
5 Critère central de définition du contrat de travail, «le lien de subordination est caractérisé par l'exécution d'un travail sous l'autorité d'un employeur qui a le pouvoir de donner des ordres et des directives, d'en contrôler l'exécution et de sanctionner les manquements de son subordonné » (Cas. Soc., 13 novembre 1996, nº 94-13187).

6 Fleur Laronze (2013) souligne, par exemple, que le principe de non-discrimination peut être mobilisé pour renforcer la liberté d'expression des croyances religieuses ou, au contraire, justifier sa restriction. Dans la même veine, Antoine Lyon-Caen (2013: 53) considère que l'interdiction de la discrimination est « un outil, un instrument auxiliaire de la protection des libertés».

7 À l'inverse du droit européen dans lequel «la liste des motifs de discrimination n'est pas limitative, mais simplement énumérative: cela découle du texte lui-même avec l'adverbe 'notamment' » (Renucci, 2017: 134) (cf. art. 14 de la Convention européenne des droits de l'Homme).

8 Cette notion s'applique aux athées, agnostiques, sceptiques et indifférents (CEDH, 25 mai 1993, n 14307/88), aux convictions philosophiques, etc.

9 Rappelons que l'article 1103 du Code civil décrète que: "Les contrats légalement formés tiennent lieu de loi à ceux qui les ont faits». La liberté contractuelle est, par définition, "la faculté de s'engager de son plein gré vis-à-vis d'autrui à faire quelque chose à quoi on pourra être ultérieurement contraint si on refuse de s'exécuter volontairement» (Fabre-Magnan, 2018: 148).

10 Objet particulièrement difficile à envisager juridiquement (Souvignet, 2017), le prosélytisme peut se définir comme un dépassement ou un abus du droit d'expression et une tentative d'imposer à autrui ses idées et ses convictions en usant d'une pression morale, d'actes de violence ou de harcèlement (Casado, 2017).

11 En ce sens, CEDH, 15 janvier 2013, Affaire Eweida et autres c/ Royaume-Uni, requêtes n 48420/10, 59842/10, 51671/10 et 36516/10.

12 Acte réglementaire de droit privé, «le règlement intérieur est un document unilatéral par lequel le chef d'entreprise fixe unilatéralement les règles d'organisation et de discipline, les normes d'hygiène et de sécurité applicables sur les lieux de travail ainsi que les droits de la défense du salarié et certaines dispositions sur le harcèlement sexuel et le harcèlement moral » (Favennec-Héry et Verkindt, 2018: 39).

13 Rappelons que le règlement intérieur n'est obligatoire que dans les entreprises dont l'effectif est supérieur ou égal à 20 salariés pendant une période de six mois. II reste facultatif dans les entreprises de moins de 20 salariés. À partir de 2020, en vertu de la récente promulgation de la LOi PACTE, le seuil d'effectif rendant obligatoire la mise en place d'un règlement intérieur passera de 20 à 50 salariés.

14 Cet article stipule: "La liberté de manifester sa religion ou ses convictions ne peut faire l'objet d'autres restrictions que celles qui, prévues par la loi, constituent des mesures nécessaires, dans une société démocratique, à la sécurité publique, à la protection de l'ordre, de la santé ou de la morale publiques, ou à la protection des droits et libertés d'autrui ».

15 Rappelons tout de même que la très large majorité du contentieux juridique contemporain en France sur l'expression du fait religieux dans l'entreprise concerne des femmes de confession musulmane. 


\section{Bibliographie}

Adam, Patrice, Martine Le Friant, Laurence Pécaut-Rivolier et Yasmine Tarasewicz (2016) «La religion dans l'entreprise: L'art difficile des limites», Revue de droit du travail, 9, 532-541.

Altwegg-Boussac, Manon (2011) «L'évolution des rapports entre la liberté d'entreprendre et les droits sociaux devant le Conseil constitutionnel », dans V. Champell-Desplats et D. Lochak (dir.), Libertés économiques et droits de l'Homme, Paris: Presses universitaires de Paris ouest, p. 245-256.

Antonmattei, Paul-Henri (2014) «À propos de la liberté religieuse dans l'entreprise», Revue de droit du travail, 6, 391-396.

Auzero, Gilles, Dirk Baugard et Emmanuel Dockès (2018) Droit du travail, Paris: éditions Dalloz, 1650 pages.

Avout $\left(d^{\prime}\right)$, Louis (2014) «La liberté d'entreprendre au bûcher? Retour sur une critique récente de la jurisprudence du Conseil constitutionnel », Recueil Dalloz, n²2, 1287-1292.

Barth, Isabelle, coord. (2012) Management et religions: Décryptage d'un lien indéfectible, Paris: éditions EMS, 291 pages.

Baudérot, Jean (2015) Les 7 laïcités françaises: Le modèle français de laïcité n'existe pas, Paris: éditions de la Maison des sciences de l'Homme, 175 pages.

Bereni, Laure et Vincent-Arnaud Chappe (2011) «La discrimination, de la qualification juridique à l'outil sociologique», Politix, 24 (94), 9-34.

Bobineau, Olivier (2012) «La spécificité du régime français de laïcité: grilles de lecture à partir des sciences humaines », Revue d'éthique et de théologie morale, 2 (269), 49-74.

Calvès, Gwénaële (2018) «Le critère de 'religion ou convictions', même sens et même portée à Luxembourg et à Strasbourg?», Droit social, 4, 323-329.

Casado, Arnaud (2017) «Prosélytisme dans l'entreprise de droit privé ne gérant pas un service public en droit du travail », Société, droit et religion, 1 (7), 35-52.

Cesaro, Jean-François (2009) «La subordination», dans B. Teyssié (dir.), Les notions fondamentales en droit du travail, Paris: éditions Panthéon Assas, p. 129-147.

Champeil-Desplats, Véronique (2007) "La liberté d'entreprendre au pays des droits fondamentaux», Droit social, 1, 19-27.

Cœuret, Alain, Élisabeth Fortis et François Duquesne (2016) Droit pénal du travail, Paris: LexisNexis, 611 pages.

Drago, Guillaume et Martine Lombard (2003) Les libertés économiques, Paris : éditions PanthéonAssas, 169 pages.

Duchange, Grégoire (2014) Le concept d'entreprise en droit du travail, Paris : éditions LexisNexis, 241 pages.

Fabre, Alexandre (2012) Le régime du pouvoir de l'employeur, éditions LGDJ Lextenso éditions, 420 pages.

Fabre-Magnan, Muriel (2018) L'institution de la liberté, Paris: Presses Universitaires de France (PUF), 349 pages.

Favennec-Héry, Françoise, dir. (2013) La sécurité juridique en droit du travail, Paris: éditions LexisNexis, 132 pages. 
Favennec-Héry, Françoise et Pierre-Yves Verkindt (2018) Droit du travail, LGDJ Lextenso éditions, 708 pages.

Favoreau, Louis, Patrick Gaïa, Richard Ghevontian, Ferdinand Mélin-Soucramanien, Annabelle Pena, Otto Pfersmann, Joseph Pini, André Roux, Guy Soffoni et Jérôme Tremeau (2015) Droit des libertés fondamentales, Paris: éditions Dalloz, 728 pages.

François, Gwennhaël (2013) «Religion et état de santé du salarié », Semaine sociale Lamy, 1611, 23-26.

Galindo, Géraldine et Joëlle Surply (2013) «Quel processus d'apprentissage de la gestion du fait religieux dans les entreprises françaises », Management international, 17 (numéro horssérie), 37-49.

Galindo, Géraldine et Hédia Zannad (2014) «Les grandes entreprises françaises et la religion: Proposition d'une grille d'analyse pour décrypter les postures adoptées», Revue interdisciplinaire Management, Homme et Entreprise, 4 (13), 40-53.

Gardes, Delphine (2017) «La liberté religieuse du travailleur salarié », Empan, 4 (108), 98-105.

Gaudu, François (2010) «La religion dans l'entreprise», Droit social, 1, 65-71.

Gherardi, Sophie (2017) «Le fait religieux en entreprise, un fait social total», Études, 12, 75-86.

Gratton, Laurène (2012) "Volonté du salarié et droits fondamentaux», dans T. Sachs (dir.), La volonté du salarié, Paris: éditions Dalloz, p. 183-197.

Gonzalez, Gérard (2012) «Liberté de pensée, de conscience et de religion », J. Andriantsimbazovina et al. (dir.), Dictionnaire des droits de l'Homme, Paris: éditions PUF, p. 636-639.

Gonzalez, Gérard (2006) "L'exigence de neutralité des services publics», G. Gonzalez (dir.), Laïcité, liberté de religion et Convention européenne des droits de l'Homme, Bruxelles: éditions Nemesis, Bruylant, p. 153-200.

Hennette Vauchez, Stephanie et Vincent Valentin (2014) L'affaire Baby Loup ou la nouvelle laïcité, Paris: LGDJ Lextenso éditions, 116 pages.

Hennette-Vauchez, Stephanie et Cyril Wolmark (2016) «Plus vous discriminez, moins vous discriminez: À propos des conclusions de l'avocate générale dans l'affaire sur le port du voile au travail, CJUE, Achbita, aff. C. 157-15», Semaine sociale Lamy, octobre, 1739, 25-29.

Hervieu, Nicolas (2011) "Droits de l'Homme et libertés économiques devant les juges européens: I'illusion d'une harmonie », dans V. Champell-Desplats et D. Lochak (dir.), Libertés économiques et droits de l'Homme, Paris: Presses universitaires de Paris ouest, p. 215-228.

Laronze, Fleur (2013) «L'articulation de la liberté de religion et du principe de non-discrimination CEDH 15 janvier 2013, aff. 48420/10, 59842/10, 51671/10, 36516/10», Revue de droit du travail, 5, 337-338.

Lebreton, Gilles (2012) «Liberté», dans J. Andriantsimbazovina et al. (dir.), Dictionnaire des droits de l'Homme, Paris: PUF, p. 619-622.

Lécuyer, Yannick (2016) Régimes des libertés et droits fondamentaux, Paris: Gualino éditeur, 48 pages.

Lokiec, Pascal (2012) «Refuser», dans T. Sachs (dir.), La volonté du salarié, Paris: éditions Dalloz, p. 103-112.

Lalouette, Jacqueline (2005) La séparation des Églises et de l'État: genèse et développement d'une idée, 1789-1905, Paris: éditions du Seuil, 446 pages. 
Lombard, Martine (2012) «Liberté d'entreprise et libertés économiques», dans J. Andriantsimbazovina et al. (dir.), Dictionnaire des droits de l'Homme, Paris: PUF, p. 625-628.

Lyon-Caen, Antoine (2013) "Variations sur la discrimination ou le pluriel derrière le singulier», dans G. Borenfreund et I. Vacarie (dir.), Le droit social, l'égalité et les discriminations, Paris: éditions Dalloz, p. 51-60.

Lyon-Caen, Antoine (2002) «Le droit du travail et la liberté d'entreprendre», Droit social, 3, 258-263.

Machelon, Jean-Pierre (2015) «Combats d'hier, laïcité d'aujourd'hui: retour sur la Loi du 9 décembre 1905 », Le Débat, 3 (185), 83-94.

Maggi-Germain, Nicole (2015) «Communauté, communautarisme, religion et fait religieux: de la nécessité d'opérer quelques clarifications sémantiques et juridiques », Droit social, 9, 674-681.

Manigot, Vincent (2012) Le traitement juridique de la discrimination en entreprise: Réflexions sur un risque, Paris: éditions LexisNexis, 385 pages.

Mazeau, Antoine (2016) Droit du travail, Paris: Montchrestien Lextenso éditions, 688 pages.

Moreau, Marie-Ange (2002) «Les justifications des discriminations», Droit social, 12, 11121124.

Morena, Frédérique de la (2015) «Du principe de laïcité républicaine à l'application des valeurs de laïcité dans l'entreprise », Droit social, 9, 699-707.

Muzny, Petr (2012) «Proportionnalité (principe de - et contrôle de -)», dans J. Andriantsimbazovina et al. (dir.), Dictionnaire des droits de l'Homme, Paris: PUF, p. 810-814.

Pagnerre, Yannick (2017) «[Liberté de] Religion vs [Liberté d']Entreprise», Droit social, 5, 450462.

Pagnerre, Yannick (2016) «Principe de neutralité dans l'entreprise », Droit social, 11, 880-885.

Philip-Gay, Mathilde (2016) Droit de la laïcité, Paris: éditions Ellipse, 288 pages.

Porta, Jérôme (2013) «Non-discrimination, égalité et égalité de traitement - À propos des sens de l'égalité», dans G. Borenfreund et I. Vacarie (dir.), Le droit social, l'égalité et les discriminations, Paris: éditions Dalloz, p. 9-38.

Poulat, Émile (2003) Notre laïcité publique: «La France est une République laïque», Paris: Berg International éditeurs, 435 pages.

Ray, Jean-Emmanuel (2009) «Les libertés dans l'entreprise », Pouvoirs, (3) 130, 127-142.

Renucci, Jean-François (2017) Droit européen des droits de l'Homme: droits et libertés fondamentaux garantis par la CEDH, Paris: éditions Dalloz, 552 pages.

Sabete, Wagdi (2012) "Limitation aux droits», dans J. Andriantsimbazovina et al. (dir.), Dictionnaire des droits de l'Homme, Paris: PUF, p. 656-662.

Sachs, Tatiana, dir. (2012) La volonté du salarié, Paris: éditions Dalloz, 282 pages.

Santoro, Guillaume (2010) "L'évolution du principe de liberté d'établissement en droit communautaire: un mouvement de libéralisation depuis l'arrêt Cartesio», Revue internationale de droit économique, 24 (3), 351-372.

Schütz, Gabrielle (2018) Jeunes, jolies et sous-traitées: les hôtesses d'accueil, Paris: éditions La Dispute, 224 pages. 
Shelton, Dinah, ed. (2015) The Oxford Handbook of International Human Rights Law, Oxford: Oxford University Press, 1018 pages.

Souvignet, Xavier (2017) «Prosélytisme et Cour européenne des droits de l'Homme: Prosélytisme de la Cour européenne des droits de l'Homme? », Société, droit et religion, 1 (7), 53-70.

Waquet, Philippe, Yves Struillou et Laurence Pécaut-Rivolier (2014) Pouvoirs du chef d'entreprise et libertés du salarié: Du salarié-citoyen au citoyen-salarié, Paris: éditions Liaisons, 340 pages.

Weave, Gary R. et Bradley Agle (2002) «Religion in the Workplace: Reaffirming Complexity », The Academy of Management Review, 27 (4), 502-504.

Willaime, Jean-Paul (2005) «1905 et la pratique d'une laïcité de reconnaissance sociale des religions », Archives de sciences sociales des religions, 129, 67-82.

Yamane, David,ed. (2017) Handbook of Religion and Society, Switzerland: Springer International Publishing AG, 568 pages.

Zuber, Valentine (2017) La laïcité en débat au-delà des idées reçues, Paris: Le Cavalier Bleu éditions, 190 pages.

\section{RÉSUMÉ}

\section{Analyse juridique de la liberté de religion dans les entreprises françaises: entre protection du pouvoir de l'employeur et défense de la volonté du salarié}

Dans un monde globalisé marqué par le multiculturalisme et le pluralisme confessionnel, la place du religieux cristallise les débats médiatiques, politiques, intellectuels et juridiques dans beaucoup de pays occidentaux. Sur fond de difficultés d'intégration professionnelle de personnes de confession musulmane et de discriminations religieuses au travail, la question du fait religieux s'est invitée dans les entreprises où les manifestations des convictions religieuses se sont diversifiées et complexifiées. L'analyse de ce phénomène social se caractérise par une diversité d'approches et d'angles de vue. Elle illustre tout à la fois la richesse, mais aussi la complexité des enjeux soulevés par les études du lien entre religion et management.

Cet article vise à en proposer une lecture juridique souvent absente dans la littérature en sciences de gestion. Nous chercherons à montrer que la régulation juridique de l'expression des convictions religieuses dans l'entreprise procède d'un champ de tension en équilibre instable entre les normes de droit qui, d'une part, protègent les droits et libertés de religion des salariés et celles, d'autre part, limitent l'exercice du pouvoir de l'employeur. La logique juridique associée à la prise en considération du religieux procède ainsi de la recherche d'une conciliation entre ces deux blocs de droits et libertés. Cette combinaison se nourrit du caractère incertain et équivoque d'un cadre juridique dans lequel les arbitrages entre des prétentions juridiques contradictoires ne procèdent pas d'une application mécanique de règles abstraites et impersonnelles. En effet, il n'existe pas de limites 
précises et univoques à la manifestation des croyances et des convictions religieuses dans l'entreprise. Leur expression exige toujours des réponses équilibrées.

MOTS-CLÉS: fait religieux, liberté de religion, liberté d'entreprendre, principe de neutralité.

\section{SUMMARY}

\section{Legal Analysis of the Freedom of Religion in French Companies: Between Protecting Employer Power and the Wishes of the Employee}

In a globalized world characterized by multiculturalism and religious pluralism, the place of religion crystallizes media, political, intellectual and legal debates in many Western countries. Against the backdrop of difficulties related to the professional integration of people of the Muslim faith and religious discrimination at work, the issue of religion has arisen in those companies in which the expression of religious beliefs has diversified and become more complex. The analysis of this social phenomenon is characterized by a diversity of approaches and perspectives. It illustrates both the richness and complexity of the issues raised by studies of the link between religion and management.

This article aims to provide a legal interpretation that is often absent in management science literature. We seek to show that legal regulation of the expression of religious beliefs in a company is based on an unstable balance of tension between the legal norms which, on the one hand, protect the religious rights and freedoms of employees and those which, on the other, limit the exercise of the employer's power. The legal logic associated with religious considerations thus stems from the search for conciliation between these two blocks of rights and freedoms. This combination is fuelled by the uncertain and ambiguous nature of a legal framework in which arbitration between conflicting legal claims is not based on the mechanical application of abstract and impersonal rules. Indeed, there are no precise and unambiguous limits to the manifestation of religious beliefs and convictions in a company. Their expression always requires balanced responses.

KEYWORDS: religion, freedom of religion, entrepreneurial freedom, principle of neutrality.

\section{RESUMEN}

Análisis jurídico de la libertad de religión en las empresas francesas: entre protección del poder del empleador y defensa de la voluntad del empleado

En un mundo globalizado marcado por el multiculturalismo y el pluralismo confesional, el lugar de la religión cristaliza los debates mediáticos, políticos, intelec- 
tuales y jurídicos en muchos países occidentales. En el contexto de las dificultades de integración profesional de las personas de convicción musulmana y de discriminación religiosa en el trabajo, la cuestión de la religión se ha introducido en las empresas donde las manifestaciones de convicciones religiosas se han diversificado y se han vuelto más complejas. El análisis de este fenómeno social se caracteriza por una diversidad de enfoques y puntos de vista. Esto ilustra a la vez la riqueza y la complejidad de las cuestiones planteadas por los estudios del vínculo entre religión y gerencia.

Este artículo propone una lectura jurídica a menudo ausente en la literatura en ciencias de la gestión. Buscaremos demostrar que la regulación jurídica de la expresión de las convicciones religiosas en la empresa procede de un campo de tensión en equilibrio inestable entre las normas de derecho que, por un lado, protegen los derechos y libertades de religión de los empleados, y por otro lado, aquellas que limitan el ejercicio del poder del empleador. La lógica jurídica asociada a la consideración de la religión procede de la búsqueda de una conciliación entre estos dos bloques de derechos y libertades. Esta combinación se alimenta de la naturaleza incierta y equívoca de un marco jurídico en el que los arbitrajes entre pretensiones jurídicas contradictorias no proceden de una aplicación mecánica de reglas abstractas e impersonales. Por cierto no existen límites precisos y unívocos a la manifestación de creencias y convicciones religiosas en la empresa. Su expresión siempre exige respuestas equilibradas.

PALABRAS CLAVES: hecho religioso, libertad de religión, libertas de empresa, principio de neutralidad. 